\title{
Model Predictive Control for Discrete Event Systems with Partial Synchronization
}

\author{
Xavier David-Henriet ${ }^{\mathrm{a}, \mathrm{b}, \mathrm{c}}$, Laurent Hardouin ${ }^{\mathrm{b}}$, Jörg Raisch ${ }^{\mathrm{a}, \mathrm{c}}$, \\ Bertrand Cottenceau ${ }^{b}$ \\ ${ }^{a}$ Technische Universität Berlin, Fachgebiet Regelungssysteme, 10587 Berlin, Germany \\ ${ }^{\mathrm{b}}$ Laboratoire Angevin de Recherche en Ingénierie des Systèmes, LARIS, ISTIA, \\ Université d'Angers, Angers 49000, France \\ ${ }^{\mathrm{c}}$ Max-Planck-Institut für Dynamik komplexer technischer Systeme, \\ Fachgruppe System- und Regelungstheorie, 39106 Magdeburg, Germany
}

\begin{abstract}
In this paper, we consider discrete event systems divided in a main system and a secondary system such that the inner dynamics of each system is ruled by standard synchronizations and the interactions between both systems are expressed by partial synchronizations (i.e., event $e_{2}$ can only occur when, not after, event $e_{1}$ occurs) of events in the secondary system by events in the main system. The main contribution consists in adapting model predictive control, developed in the literature for $(\max ,+)$-linear systems, to the considered class of systems. This problem is solved under the condition that the performance of the main system is never degraded to improve the performance of the secondary system. Then, the optimal input is selected to respect the output reference and the remaining degrees of freedom are used to ensure just-in-time behavior. The unconstrained problem is solved in linear time with respect to the length of the prediction horizon.
\end{abstract}

Key words: Discrete event systems, synchronization, model-based control, predictive control, transportation control, timed Petri nets

\section{Introduction}

A discrete event system is a dynamical system driven by the instantaneous occurrence of events. In the following, we focus on a particular class of time-driven (i.e., the occurrence of events is only possible at clock ticks) discrete event systems, the dynamics of which is defined by synchronization rules. The standard synchronization corresponds to the following condition: occurrence $k$ of event $e_{1}$ is at least $\tau$ units of time after occurrence $k-l$ of event $e_{2}$. Discrete event systems ruled only by standard synchronizations, called ( $\max ,+$ )-linear systems, have been widely studied and admit linear state-space representations in suitable algebraic structures such as the $(\max ,+)$-algebra and the $(\min ,+)$-algebra [8]. During

Email addresses:

xavier.david-henriet@control.tu-berlin.de (Xavier David-Henriet), laurent.hardouin@univ-angers.fr (Laurent Hardouin), raisch@control.tu-berlin.de (Jörg Raisch), bertrand.cottenceau@univ-angers.fr (Bertrand Cottenceau). the last two decades, a rich control theory has been developed for this class of systems (e.g., optimal feedforward control [2], model predictive control (MPC) [6,9], and model reference control [3]). Other synchronization rules have recently been investigated such as soft synchronization [7] and partial synchronization [4] (i.e., event $e_{2}$ can only occur when event $e_{1}$ occurs). Partial synchronization is useful when a system $A$ is offering a service for a time window to another system $B$. For example, in a public transportation network, a passenger can access a train only when the train is at the train station or a car can cross an intersection only when, not after, the traffic light is green. In this paper, we assume that the inner dynamics of systems $A$ and $B$ are driven by standard synchronizations and that system $A$ is not affected by system $B$. Then, the complete system is divided in a main system and a secondary system (with disjoint event sets) such that the interactions between both systems are expressed by partial synchronizations of events in the secondary system by events in the main system. We focus on the control of this kind of systems under the assumption that the performance of the main 
system is never degraded to improve the performance of the secondary system. This makes sense in many applications, where the main system is not only used by the secondary system, but shared by many users. For example, a train (main system) does not wait for delayed passengers (secondary system). We consider MPC, initially introduced for $(\max ,+)$-linear systems in $[6]$. The unconstrained case is investigated in (min, + )-algebra, which is more suitable to deal with partial synchronizations than $(\max ,+)$-algebra [5]. We prove that the unconstrained MPC optimization problem is solved at each step with a complexity linear with the length of the prediction horizon.

Necessary mathematical tools are recalled in $\S 2$. In $\S 3$, the modeling is addressed and the behavior under the earliest functioning rule is investigated. In $\S 4$, the main contribution of this paper, namely MPC, is introduced. Finally, the proposed control approach is applied to a supply chain in $\S 5$.

\section{Mathematical Preliminaries}

The (min, +)-algebra, denoted $\overline{\mathbb{N}}_{\text {min }}$, is defined as the set $\mathbb{N}_{0} \cup\{+\infty\}$ endowed with min, denoted $\oplus$, and + , denoted $\otimes$. Formally, $\overline{\mathbb{N}}_{\text {min }}$ is a dioid [1]. As in standard algebra, the product $\otimes$ is often denoted by juxtaposition (i.e., $a b$ corresponds to $a \otimes b$ ). The operation $\oplus$ induces an order relation $\preceq$ on $\overline{\mathbb{N}}_{\text {min }}$ defined as

$$
\forall a, b \in \overline{\mathbb{N}}_{\text {min }}, \quad a \oplus b=b \Leftrightarrow a \preceq b
$$

Obviously, $\preceq$ corresponds to the dual of the standard order $\leq$ in $\overline{\mathbb{N}}_{0}$ (i.e., $a \succeq b \Leftrightarrow a \leq b$ ). In the following, only the order relation $\preceq$ is used for expressions related to $\overline{\mathbb{N}}_{\text {min }}$. Two particular elements in $\overline{\mathbb{N}}_{\text {min }}$ are the zero element (i.e., the neutral element of $\oplus$ ), equal to $+\infty$ and denoted $\varepsilon$, and the unit element (i.e., the neutral element of $\otimes$ ), equal to 0 and denoted $e$. By analogy with standard linear algebra, $\oplus$ and $\otimes$ are defined for matrices with entries in $\overline{\mathbb{N}}_{\text {min }}$. For $A, B \in \overline{\mathbb{N}}_{\text {min }}^{n \times m}$ and $C \in \overline{\mathbb{N}}_{\text {min }}^{m \times p}$,

$$
(A \oplus B)_{i j}=A_{i j} \oplus B_{i j} \text { and }(A \otimes C)_{i j}=\bigoplus_{k=1}^{m} A_{i k} C_{k j}
$$

The sum $\oplus$ and the product $\otimes$ are order-preserving. Two additional operations on $\overline{\mathbb{N}}_{\text {min }}$ are $\wedge$, corresponding to the max in standard algebra, and $\phi$, where $a \oint b$ denotes the greatest solution of $a \otimes x \preceq b$. An extension of these operations to the matrix case is straightforward.

\section{Input-Output Behavior}

To capture the behavior of discrete event systems, a counter $f$ is associated with the eponymous event $f$ to count its occurrences. Formally, the counter $f$ is a mapping from $\mathbb{Z}$ to $\overline{\mathbb{N}}_{\text {min }}$, where $f(t)$ is defined as the number of occurrences of event $f$ before or at time $t$. Interesting properties of counter $f$ are

$$
f(t)=e \text { for } t<0 \text { and } t_{1} \leq t_{2} \Rightarrow f\left(t_{1}\right) \succeq f\left(t_{2}\right)
$$

An advantage of the counter representation is its ability to easily express standard synchronizations in $\overline{\mathbb{N}}_{\text {min }}$. For example, the standard synchronizations "occurrence $k$ of event $e_{2}$ is at least $\tau_{1}$ units of time after occurrence $k-l_{1}$ of event $e_{1,1}$ and at least $\tau_{2}$ units of time after occurrence $k-l_{2}$ of event $e_{1,2}$ " correspond to the following inequality in $\overline{\mathbb{N}}_{\text {min }}$ :

$$
\forall t \in \mathbb{Z}, \quad e_{2}(t) \succeq l_{1} e_{1,1}\left(t-\tau_{1}\right) \oplus l_{2} e_{1,2}\left(t-\tau_{2}\right)
$$

Partial synchronization can also be expressed by a condition on counters. For example, "event $e_{2}$ can only occur when, not after, event $e_{1}$ occurs" is equivalent to "if event $e_{1}$ does not occur at time $t$, then event $e_{2}$ does not occur at time $t$ ". This corresponds to

$$
\forall t \in \mathbb{Z}, \quad e_{1}(t)=e_{1}(t-1) \Rightarrow e_{2}(t)=e_{2}(t-1)
$$

Next, the conditions induced by synchronizations on the dynamics of the main system and of the secondary system are formally described. For each system, the event set is partitioned into state, input, and output events as it is done for $(\max ,+)$-linear systems. The behavior of the main system must be a solution of

$$
\left\{\begin{array}{l}
x_{1}(t) \succeq A_{10} x_{1}(t) \oplus A_{11} x_{1}(t-1) \oplus B_{1} u_{1}(t) \\
y_{1}(t) \succeq C_{1} x_{1}(t)
\end{array}\right.
$$

where $x_{1}, u_{1}$, and $y_{1}$ respectively denote the vectors of counters associated with state, input, and output events in the main system. The entries of matrices $A_{10}, A_{11}$, $B_{1}$, and $C_{1}$ are given by the standard synchronizations in the main system. Due to partial synchronizations, the dynamics of the secondary system is slightly more complicated. The behavior of the secondary system must be a solution of

$$
\left\{\begin{array}{l}
x_{2}(t) \succeq A_{20} x_{2}(t) \oplus A_{21} x_{2}(t-1) \oplus B_{2} u_{2}(t) \\
y_{2}(t) \succeq C_{2} x_{2}(t) \\
\forall i,\left(\exists x_{1, j} \in \mathcal{S}_{i} \mid x_{1, j}(t)=x_{1, j}(t-1)\right) \\
\quad \Rightarrow x_{2, i}(t)=x_{2, i}(t-1)
\end{array}\right.
$$

where $x_{2}, u_{2}$, and $y_{2}$ respectively denote the vectors of counters associated with state, input, and output events in the secondary system. The entries of matrices $A_{20}$, $A_{21}, B_{2}$, and $C_{2}$ are given by the standard synchronizations in the secondary system. The third condition expresses partial synchronizations: $\mathcal{S}_{i}$ denotes the set 
of state events in the main system synchronizing state event $x_{2, i}$ in the secondary system.

Remark 1 By analogy with (max, +)-linear systems, we assume that matrices $A_{10}$ and $A_{20}$ are strictly lower triangular [1].

Apart from the partial synchronization condition, the main and the secondary system are described by the same model structure. This allows us to treat both systems in a unified way as follows.

$$
\left\{\begin{array}{l}
x(t) \succeq A_{0} x(t) \oplus A_{1} x(t-1) \oplus B u(t) \\
y(t) \succeq C x(t) \\
\forall i, \quad \alpha_{i}(t)=0 \Rightarrow x_{i}(t)=x_{i}(t-1)
\end{array}\right.
$$

where $x, u$, and $y$ are vectors (with dimension $n, p$, and $q$ ) of counters associated with state, input, and output events. Mapping $\alpha_{i}$ is defined from $\mathbb{Z}$ to $\{0,1\}$ such that $\alpha_{i}(t)=1$ if, and only if, partial synchronizations allow event $x_{i}$ to occur at time $t$. For an event $x_{i}$ of the main system, $\alpha_{i}(t)=1$. For an event $x_{i}$ of the secondary system,

$$
\alpha_{i}(t)=\left\{\begin{array}{l}
0 \text { if } \exists x_{1, j} \in \mathcal{S}_{i} \mid x_{1, j}(t)=x_{1, j}(t-1) \\
1 \text { otherwise }
\end{array}\right.
$$

Problem (1) only describes admissible behaviors. In the following, a standard behavior is introduced, namely the behavior under the earliest functioning rule (i.e., each state or output event occurs as soon as possible). Hence, assuming that the behavior is known for times $\tau<t$, the behavior at time $t$ is given by the least solution $(y(t), x(t))$ of $(1)$ with the additional causality conditions $y(t) \preceq y(t-1)$ and $x(t) \preceq x(t-1)$. Checking existence and uniqueness of the behavior under the earliest functioning rule is not difficult and leads to the following input-output behavior:

$$
\left\{\begin{array}{l}
x(t)=H(x(t-1), u(t), t) \\
y(t)=C x(t)
\end{array}\right.
$$

where

$$
\begin{aligned}
& H(x(t-1), u(t), t)_{i}= \\
& \left\{\begin{array}{l}
x_{i}(t-1) \text { if } \alpha_{i}(t)=0 \\
\bigoplus_{j=1}^{i-1} A_{0, i j} H(x(t-1), u(t), t)_{j} \\
\oplus\left(A_{1} x(t-1) \oplus B u(t)\right)_{i} \text { if } \alpha_{i}(t)=1
\end{array}\right.
\end{aligned}
$$

The previous discussion ensures the existence and uniqueness of the behavior under the earliest functioning rule for the considered systems and leads to an algorithm to compute it. First, the behavior of the main system is obtained with $\alpha_{i}(t)=1$. Then, the mappings $\alpha$ associated with the secondary system are given by (2). Finally, the behavior of the secondary system is computed.

\section{Model Predictive Control}

Model predictive control (MPC) for $(\max ,+)$-linear systems has been investigated in $[6,9]$. In the following, this approach is adapted to the considered class of systems. Let us briefly recall the principle of MPC. From time $t$ to $t+1$, an optimal input is computed over a prediction horizon by minimizing a cost function using a prediction of the future behavior based on the model. At time $t+1$, this optimal input is applied to the system, the prediction horizon is shifted by one time step, and the computation is repeated using event occurrences at time $t+1$. The considered control approach gives priority to the main system over the secondary system: the performance of the main system is never degraded to improve the performance of the secondary system. This allows us to first compute optimal inputs for the main system. If a single optimal input for the main system exists, it remains to calculate the optimal input for the secondary system under a predefined behavior of the main system. The cost criterion used in the following leads to a single optimal input, which allows us to separate the complete problem in two subproblems.

In the following, a method to obtain the optimal input for a system described by (3) is presented. The optimal input is selected to minimize the expected delay with respect to an output reference. The remaining degrees of freedom in the choice of the optimal input are used to ensure the just-in-time behavior (i.e., input events occur as late as possible). Notice that this approach suits transportation problems, as minimizing the expected delay corresponds to respecting the schedule as closely as possible and just-in-time behavior reduces the travel time. The cost function used to minimize the expected delay at time $t+1$ is

$$
J_{1}(\tilde{y})=\sum_{\tau=t+1}^{t+T} \sum_{j=1}^{q} \max \left(r_{j}(\tau)-\tilde{y}_{j}(\tau), 0\right)
$$

where $\tilde{y}$ is the predicted output, $r$ is the output reference, and $T$ is the length of the prediction horizon. This cost function is the equivalent for counters of the tardiness criterion introduced in [6] for daters. If $J_{1}(\tilde{y})=$ 0 , the predicted output respects the output reference (i.e., $\tilde{y}(\tau) \preceq r(\tau))$. The cost function used to ensure the just-in-time behavior at time $t+1$ is

$$
J_{2}(\tilde{u})=\sum_{\tau=t+1}^{t+T} \sum_{j=1}^{p}\left(\tilde{u}_{j}(\tau)-u_{j}(t)\right)
$$


If $J_{2}(\tilde{u})=0$, no input events occur over the prediction horizon. The optimization problem to solve between time $t$ and time $t+1$ is

$$
\begin{aligned}
& \underset{\tilde{u}(t+1), \ldots, \tilde{u}(t+T)}{\operatorname{minimize}}\left(J_{1}(\tilde{y}), J_{2}(\tilde{u})\right) \\
& \text { subject to } \\
& \left\{\begin{array}{l}
\tilde{x}(\tau)=H(\tilde{x}(\tau-1), \tilde{u}(\tau), \tau) \\
\tilde{y}(\tau)=C \tilde{x}(\tau) \\
\tilde{u}(\tau) \preceq \tilde{u}(\tau-1)
\end{array}\right. \\
& \text { for } t+1 \leq \tau \leq t+T \\
& \tilde{u}(t)=u(t) \text { and } \tilde{x}(t)=x(t)
\end{aligned}
$$

The subscript $\mathcal{L}$ means that the considered order relation for the cost function with values in $\mathbb{R}^{2}$ is the lexicographic order relation. Before solving (5), an intermediate lemma is introduced.

Lemma 2 The inequality $x(t)=H(x(t-1), u(t), t) \preceq$ $z(t)$ admits a greatest solution $(x(t-1), u(t))$, denoted $(F(z(t), t), G(z(t), t))$.

PROOF. Let us denote $\mathcal{K}=\left\{j \mid \alpha_{j}(t)=1\right\}$ and $\mathcal{K}_{i}=\left\{j \mid j>i\right.$ and $\left.\alpha_{j}(t)=1\right\}$. Then, according to (4), the least upper bound $\zeta(t)$ of $x(t)$ is given by

$$
\zeta_{i}(t)=z_{i}(t) \wedge \bigwedge_{j \in \mathcal{K}_{i}} A_{0, j i} \phi \zeta_{j}(t)
$$

Based on $\zeta(t)$, the greatest solution $(F(z(t), t), G(z(t), t))$ is computed using (4). This leads to

$$
\begin{aligned}
F(z(t), t)_{i} & =\left\{\begin{array}{l}
\zeta_{i}(t) \wedge \bigwedge_{j \in \mathcal{K}} A_{1, j i} \phi \zeta_{j}(t) \text { if } \alpha_{i}(t)=0 \\
\bigwedge_{j \in \mathcal{K}} A_{1, j i} \phi \zeta_{j}(t) \text { if } \alpha_{i}(t)=1
\end{array}\right. \\
G(z(t), t)_{i} & =\bigwedge_{j \in \mathcal{K}} B_{j i} \phi \zeta_{j}(t)
\end{aligned}
$$

Based on Lem. 2, a solution of (5) is obtained in the following theorem when the output reference $r$ takes values in $\mathbb{N}_{0}$ over the prediction horizon (i.e., $r_{j}(\tau) \neq \varepsilon$ for $t+1 \leq \tau \leq t+T)$. In practice, this is not restrictive, as the number of event occurrences remains bounded.

Theorem 3 Denote $\tilde{y}_{\varepsilon}$ the output induced by the input $\tilde{u}_{\varepsilon}$, defined by $\tilde{u}_{\varepsilon}(\tau)=\varepsilon$ for $t+1 \leq \tau \leq t+T$ and assume that $r_{j}(\tau) \in \mathbb{N}_{0}$ for $t+1 \leq \tau \leq t+T$. Then, the unique solution of (5), denoted $\tilde{u}_{\text {opt }}$, is given by

$$
\tilde{u}_{\text {opt }}(\tau)=\bigwedge_{\tau \geq j \geq t} \tilde{v}(j) \text { for } t+1 \leq \tau \leq t+T
$$

where $\tilde{v}(t)=u(t)$ and the sequence $(\tilde{v}(\tau))_{t+1 \leq \tau \leq t+T}$ is defined by

$$
\begin{aligned}
& \zeta(t+T)=\tilde{z}(t+T) \\
& \left\{\begin{array}{l}
\tilde{v}(\tau)=G(\zeta(\tau), \tau) \\
\zeta(\tau-1)=F(\zeta(\tau), \tau) \wedge \tilde{z}(\tau-1)
\end{array}\right.
\end{aligned}
$$

where $\tilde{z}(\tau)=C \phi\left(r(\tau) \oplus \tilde{y}_{\varepsilon}(\tau)\right)$ for $t+1 \leq \tau \leq t+\tau$.

PROOF. The following optimization problem is first considered.

$$
\begin{aligned}
& \underset{\tilde{u}(t+1), \ldots, \tilde{u}(t+T)}{\operatorname{minimize}} J_{1}(\tilde{y}) \\
& \text { subject to } \\
& \qquad \begin{array}{l}
\tilde{x}(\tau)=H(\tilde{x}(\tau-1), \tilde{u}(\tau), \tau) \\
\tilde{y}(\tau)=C \tilde{x}(\tau) \\
\tilde{u}(\tau) \preceq \tilde{u}(\tau-1)
\end{array} \\
& \text { for } t+1 \leq \tau \leq t+T \\
& \tilde{u}(t)=u(t) \text { and } \tilde{x}(t)=x(t)
\end{aligned}
$$

The cost function $J_{1}$ is order-preserving: $\tilde{y}_{1} \succeq \tilde{y}_{2}$ implies $J_{1}\left(\tilde{y}_{1}\right) \geq J_{1}\left(\tilde{y}_{2}\right)$. Therefore, to solve (7), it is sufficient to find the least predicted output $\tilde{y}$, if it exists. As the product $\otimes$ is order-preserving, it is sufficient to obtain the least predicted state $\tilde{x}$, if it exists. As the mapping $H$ is order-preserving with respect to the first two arguments, it is sufficient to find the least future input $\tilde{u}$, which exists and is equal to $\tilde{u}_{\varepsilon}$. Hence, the optimal cost for $(7)$ is $J_{1}\left(\tilde{y}_{\varepsilon}\right)$, where $\tilde{y}_{\varepsilon}$ is the output induced by $\tilde{u}_{\varepsilon}$. As a lexicographic order relation is considered in (5), solving (5) is equivalent to solving the following optimization problem.

$$
\begin{aligned}
& \underset{\tilde{u}(t+1), \ldots, \tilde{u}(t+T)}{\operatorname{minimize}} J_{2}(\tilde{u}) \\
& \text { subject to } \\
& \left\{\begin{array}{l}
\tilde{x}(\tau)=H(\tilde{x}(\tau-1), \tilde{u}(\tau), \tau) \\
\tilde{y}(\tau)=C \tilde{x}(\tau) \\
\tilde{u}(\tau) \preceq \tilde{u}(\tau-1)
\end{array}\right. \\
& \text { for } t+1 \leq \tau \leq t+T \\
& J_{1}(\tilde{y})=J_{1}\left(\tilde{y}_{\varepsilon}\right) \\
& \tilde{u}(t)=u(t) \text { and } \tilde{x}(t)=x(t)
\end{aligned}
$$

Next, we show that $J_{1}(\tilde{y})=J_{1}\left(\tilde{y}_{\varepsilon}\right)$ is equivalent to $\tilde{y}(\tau) \preceq r(\tau) \oplus \tilde{y}_{\varepsilon}(\tau)$ for $t+1 \leq \tau \leq t+T$. As $J_{1}(\tilde{y}) \geq$ $J_{1}\left(\tilde{y}_{\varepsilon}\right)$ and $J_{1}\left(\tilde{y}_{\varepsilon}\right)$ is finite, $J_{1}(\tilde{y})=J_{1}\left(\tilde{y}_{\varepsilon}\right)$ is equivalent to

$$
\max \left(r_{j}(\tau)-\tilde{y}_{j}(\tau), 0\right)=\max \left(r_{j}(\tau)-\tilde{y}_{\varepsilon, j}(\tau), 0\right)
$$


for all $1 \leq j \leq q$ and $t+1 \leq \tau \leq t+T$. If $\tilde{y}_{\varepsilon, j}(\tau) \geq r_{j}(\tau)$, $\max \left(r_{j}(\tau)-\tilde{y}_{j}(\tau), 0\right)=0$. This leads to

$$
\tilde{y}_{j}(\tau) \geq r_{j}(\tau)=\min \left(r_{j}(\tau), \tilde{y}_{\varepsilon, j}(\tau)\right)
$$

Otherwise, if $r_{j}(\tau)>\tilde{y}_{\varepsilon, j}(\tau), \max \left(r_{j}(\tau)-\tilde{y}_{j}(\tau), 0\right)=$ $r_{j}(\tau)-\tilde{y}_{\varepsilon, j}(\tau)$. This leads to $\tilde{y}_{j}(\tau)=\tilde{y}_{\varepsilon, j}(\tau)$ or, as $\tilde{y} \leq \tilde{y}_{\varepsilon}$, to

$$
\tilde{y}_{j}(\tau) \geq \tilde{y}_{\varepsilon, j}(\tau)=\min \left(r_{j}(\tau), \tilde{y}_{\varepsilon, j}(\tau)\right)
$$

Replacing the constraint $J_{1}(\tilde{y})=J_{1}\left(\tilde{y}_{\varepsilon}\right)$ by an equivalent inequality over $\tilde{x}$ allows us to transform (8) in

$$
\begin{aligned}
& \underset{\tilde{u}(t+1), \ldots, \tilde{u}(t+T)}{\operatorname{minimize}} J_{2}(\tilde{u}) \\
& \text { subject to } \\
& \qquad\left\{\begin{array}{l}
\tilde{x}(\tau)=H(\tilde{x}(\tau-1), \tilde{u}(\tau), \tau) \\
\tilde{x}(\tau) \preceq \tilde{z}(\tau) \\
\tilde{u}(\tau) \preceq \tilde{u}(\tau-1)
\end{array}\right. \\
& \text { for } t+1 \leq \tau \leq t+T \\
& \tilde{u}(t)=u(t) \text { and } \tilde{x}(t)=x(t)
\end{aligned}
$$

where $\tilde{z}(\tau)=C \phi\left(r(\tau) \oplus \tilde{y}_{\varepsilon}(\tau)\right)$. As $\tilde{u}_{1} \preceq \tilde{u}_{2}$ implies $J_{2}\left(\tilde{u}_{1}\right) \geq J_{2}\left(\tilde{u}_{2}\right)$, solving (9) is equivalent to find the greatest (if it exists) input $\tilde{u}$ satisfying the conditions in (9). For $t+1 \leq \tau \leq t+T, \tilde{u}(\tau) \preceq \tilde{v}(\tau)$ where $(\tilde{v}(\tau))_{t+1 \leq \tau \leq t+T}$ is the greatest solution of

$$
\begin{aligned}
& \left\{\begin{array}{l}
\tilde{x}(\tau)=H(\tilde{x}(\tau-1), \tilde{v}(\tau), \tau) \\
\tilde{x}(\tau) \preceq \tilde{z}(\tau)
\end{array}\right. \\
& \text { for } t+1 \leq \tau \leq t+T \text { and } \tilde{x}(t)=x(t)
\end{aligned}
$$

By analogy with optimal feedforward control for $(\max ,+)$-linear systems [2], $(\tilde{v}(\tau))_{t+1<\tau<t+T}$ is given by (6). Therefore, the optimal input $\left(\tilde{u}_{o p t}(\bar{\tau})\right)_{t+1<\tau<t+T}$ is the greatest decreasing sequence less than or equal to $(\tilde{v}(\tau))_{t+1 \leq \tau \leq t+T}$. Hence,

$$
\tilde{u}_{o p t}(\tau)=\bigwedge_{\tau \geq j \geq t} \tilde{v}(j) \text { with } \tilde{v}(t)=u(t)
$$

Further, as $r$ takes value in $\mathbb{N}_{0}, r(t+T)$ is finite. Hence, due to event-causality associated with standard synchronization, $\tilde{u}_{o p t}(t+T)$ is finite. Then, $\tilde{u}_{\text {opt }}$ is the unique optimal input, as, for finite inputs, $\tilde{u}_{1} \prec \tilde{u}_{2}$ implies $J_{2}\left(\tilde{u}_{2}\right)<J_{2}\left(\tilde{u}_{1}\right)$

Th. 3 provides an algorithm to implement MPC for a dynamic system described by (3). Further, the complexity of optimal input calculation at a given time $t$ is linear with the length of the prediction horizon $T$, as the calculations of $\tilde{y}_{\varepsilon}, \tilde{v}$, and $\tilde{u}_{\text {opt }}$ come down to applying a forward or backward recursive relations over the prediction horizon. Finding the optimal input for the complete system (i.e., main and secondary system) boils down to, first, compute the optimal input for the main system, second, predict the mappings $\alpha$ associated with the secondary system, and, finally, compute the optimal input for the secondary system. Therefore, the complexity of optimal input calculation at a given time $t$ for the complete system remains linear with the length of the prediction horizon.

\section{Example}

The problem considered in this example focuses on a supply chain where intermodal containers shuttle back and forth between warehouses $A_{1}$ and $B_{1}$. The supply chain is divided in three sections: a road transport section between warehouse $A_{1}$ and train station $A$, a rail transport section between train stations $A$ and $B$, and a road transport section between train station $B$ and warehouse $B_{1}$. The supply chain is drawn in Fig. 1 (where the solid loop represents the train line, the dashed loops represent the road transport, and the dotted loop represents the supply chain). In road transport, the truck/container inter-

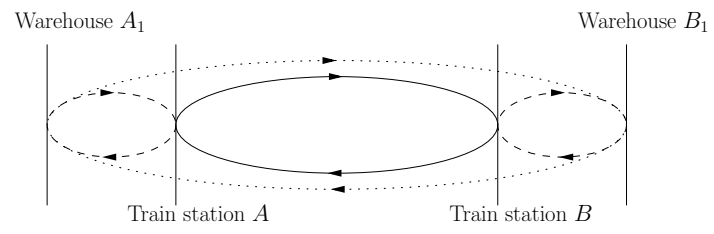

Fig. 1. The supply chain

action is neglected: sufficiently many trucks are available to deliver containers. The train/container interaction is expressed by partial synchronizations: a container leaves (resp. arrives at) a train station by train only when a train leaves (resp. arrives at) this train station. In this example, the main system corresponds to the train line and the secondary system models the transportation of the container. In Fig. 2, the overall system is represented by a timed Petri net where the dashed arrows correspond to the partial synchronizations. The proposed MPC con-

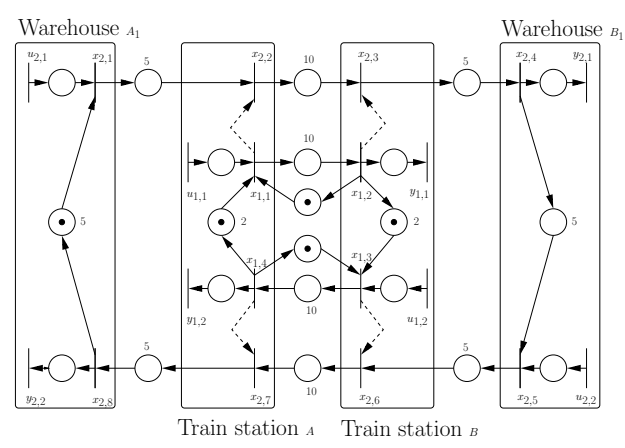

Fig. 2. Petri net representation 
trol approach is applied. As trains are shared by many users (including, but not limited to, the supply chain between warehouses $A_{1}$ and $B_{1}$ ), it makes sense to never degrade the performance of the train line to improve the performance of the supply chain between warehouses $A_{1}$ and $B_{1}$. In this example, the considered cost functions (i.e., tardiness and just-in-time criterion) are suitable to enforce delivery dates and minimize travel times. Furthermore, due to output reference update and optimal input recalculation at each time step, changes in the schedule and perturbations are taken into account.

In the following, MPC is applied to this system with a prediction horizon of length $T=100$ and the following schedules:

- a train arrives in train stations $A$ and $B$ every 15 units of time with a first arrival at $t=15$

- a container arrives in warehouse $B_{1}$ (resp. $A_{1}$ ) every 80 units of time with a first arrival at $t=40$ (resp. $t=80)$

This corresponds to the following output references:

$$
\begin{aligned}
& r_{1,1}(t)=r_{1,2}(t)=\left\lfloor\frac{t}{15}\right\rfloor \\
& r_{2,1}(t)=\left\lfloor\frac{t+40}{80}\right\rfloor \text { and } r_{2,2}(t)=\left\lfloor\frac{t}{80}\right\rfloor
\end{aligned}
$$

For the train line, the optimal input obtained with MPC is a departure from train stations $A$ and $B$ every 15 units of time with a first departure at $t=5$. Formally,

$$
u_{1,1}(t)=u_{1,2}(t)=\left\lfloor\frac{t+10}{15}\right\rfloor
$$

For the supply chain, the optimal input obtained with MPC has a more complex pattern due to interactions with the train line:

$$
\begin{aligned}
& u_{2,1}(t)=\left\lfloor\frac{t+225}{240}\right\rfloor+\left\lfloor\frac{t+150}{240}\right\rfloor+\left\lfloor\frac{t+60}{240}\right\rfloor \\
& u_{2,2}(t)=\left\lfloor\frac{t+180}{240}\right\rfloor+\left\lfloor\frac{t+105}{240}\right\rfloor+\left\lfloor\frac{t+30}{240}\right\rfloor
\end{aligned}
$$

Further, if a perturbation prevents the departure of train from train station $A$ for $50 \leq t \leq 60$, the optimal input is modified as follows:

- the train line functions at a maximal troughput (i.e., one train every 12 units of time) for $70 \leq t \leq 100$ to catch up the delay induced by the perturbation

- the first (resp. second) departure of the container from warehouse $B_{1}$ (resp. $A_{1}$ ) is delayed of 13 (resp. 15) units of time to adjust to changes in the behavior of the train line
As expected, the computation time of the optimal input at each step, experimentally observed with a Scilab implementation, is linear with the length of the prediction horizon, denoted $T$.

\begin{tabular}{|c|c|c|c|}
\hline$T$ & 128 & 256 & 512 \\
\hline Computation time (in $s$ ) & 0.36 & 0.72 & 1.42 \\
\hline
\end{tabular}

\section{Conclusion}

In this paper, we consider a particular class of discrete event systems ruled by standard and partial synchronizations. Based on a (min, +)-model, MPC is adapted to this class of systems by giving priority to the performance of the main system. In each system, the optimality criterion focuses on minimizing the expected delay, but the remaining degrees of freedom are used to ensure just-in-time behavior. At each step, the computation of the optimal input is linear with the length of the prediction horizon. As future work, we want to include a standard update for the reference output at each step to take into account additional constraints on the behavior of the system.

\section{References}

[1] F. Baccelli, G. Cohen, G. J. Olsder, and J.-P. Quadrat. Synchronization and Linearity, An Algebra for Discrete Event Systems. John Wiley and Sons, New York, USA, 1992.

[2] G. Cohen, S. Gaubert, and J. P. Quadrat. From First to Second-Order Theory of Linear Discrete Event Systems. In 12th IFAC World Congress, volume 1, pages 679-682, Sydney, Jul. 1993.

[3] B. Cottenceau, L. Hardouin, J.-L. Boimond, and J.-L. Ferrier. Model Reference Control for Timed Event Graphs in Dioids. Automatica, 37(9):1451-1458, September 2001.

[4] X. David-Henriet, L. Hardouin, J. Raisch, and B. Cottenceau. Optimal Control for Timed Event Graphs under Partial Synchronization. In 52nd IEEE Conference on Decision and Control, $C D C$ '13, pages 7609-7614, Firenze, Italy, December 2013.

[5] X. David-Henriet, L. Hardouin, J. Raisch, and B. Cottenceau. Modeling and Control for Max-Plus Systems with Partial Synchronization. In International Workshop on Discrete Event Systems, WODES'14, Paris, France, May 2014.

[6] B. De Schutter and T. J. J. van den Boom. Model Predictive Control for Max-Plus-Linear Discrete Event Systems. Automatica, 37(7):1049-1056, July 2001.

[7] B. De Schutter and T. J. J. van den Boom. MPC for Discrete-Event Systems with Soft and Hard Synchronisation Constraints. International Journal of Control, 76(1):82-94, January 2003.

[8] B. Heidergott, G. J. Olsder, and J. W. van der Woude. Max Plus at work : modeling and analysis of synchronized systems : a course on Max-Plus algebra and its applications. Princeton series in applied mathematics. Princeton University Press, Princeton (N.J.), 2006.

[9] I. Necoara. Model Predictive Control for Max-Plus-Linear and Piecewise Affine Systems. $\mathrm{PhD}$ thesis, Delft University of Technology, 2006. 